\title{
The Synthesis and Magnetic Properties of Nanosized Hematite $\left(\alpha-\mathrm{Fe}_{2} \mathrm{O}_{3}\right)$ Particles
}

\author{
T. P. Raming, ${ }^{*}$ A. J. A. Winnubst, ${ }^{*}, 1$ C. M. van Kats, $†$ and A. P. Philipse $\dagger$ \\ * Laboratory for Inorganic Materials Science, Department of Chemical Technology and MESA Research Institute, University of Twente, \\ PO Box 217, 7500 AE Enschede, The Netherlands; and †Debye Institute, van't Hoff Laboratory for Physical and Colloid Chemistry, \\ Utrecht University, Padualaan 8, 3508 CH Utrecht, The Netherlands
}

Received July 19, 2001; accepted December 20, 2001; published online April 10, 2002

The synthesis of nanosized superparamagnetic hematite particles by dissolving ferric salts in hydrochloric acid and heating at $100{ }^{\circ} \mathrm{C}$ is described. A hydrolysis reaction causes the formation of hematite particles. The influence of the sequence of additions on the resulting precipitates was studied using TEM and XRD. The magnetic behavior was characterized by magnetization measurements. It was found that small changes in the reaction conditions led to remarkable changes in final size and shape of the hematite crystallites. A well-defined subrounded morphology and an average diameter of $41 \mathrm{~nm}$ were obtained for superparamagnetic hematite particles. This is the largest size reported thus far for superparamagnetic hematite particles. () 2002 Elsevier Science (USA)

Key Words: hematite; precipitation; magnetization; superparamagnetic; crystallite size.

\section{INTRODUCTION}

Hematite $\left(\alpha-\mathrm{Fe}_{2} \mathrm{O}_{3}\right)$ has so-called parasitic or canted magnetism (1). This means that the magnetic moments of the two magnetic sublattices do not fully cancel each other, resulting in a small magnetic moment in the direction of the basal plane. When decreasing the size of magnetic particles, they change from multidomain to single domain (2). If the single-domain particles become small enough, the magnetic moment in the domain fluctuates in direction, due to thermal agitation which leads to superparamagnetism.

It is known that the coercive force opposing this effect is influenced by many factors, such as size and shape, packing density, and defects in the crystal structure. Usually, the coercive force decreases with the size of the crystallites (2). For spherical hematite it has been shown that there is a threshold diameter of about $8 \mathrm{~nm}$ below which the particles become superparamagnetic $(3,4)$. Other values for this threshold diameter have been reported as well, e.g., $14 \mathrm{~nm}$ (5).

This threshold diameter at which hematite becomes superparamagnetic was determined for particles synthesised accord-

\footnotetext{
${ }^{1}$ To whom correspondence should be addressed. Fax: xx31534894683. E-mail: a.j.a.winnubst@ct.utwente.nl.
}

ing to a precipitation developed by Matijevic and Scheiner (6), since this precipitation has been reported to produce welldefined monosized hematite crystals. This precipitation was further investigated, in order to prepare different batches of monosized hematite particles of varying particle sizes. Hematite particles of an average diameter of $41 \mathrm{~nm}$ were found to be superparamagnetic, which is the largest size reported thus far for superparamagnetic hematite particles.

\section{MATERIALS AND METHODS}

In the first method, as described earlier by Matijevic and Scheiner (6), doubly distilled water was heated to $100^{\circ} \mathrm{C}$. The water was poured into a preheated Pyrex glass beaker. The temperature remained at $100^{\circ} \mathrm{C}$. Concentrated $(37 \mathrm{wt} \%) \mathrm{HCl}$ was added to this system to make a $0.002 \mathrm{M} \mathrm{HCl}$ solution. The temperature was kept at $90-100^{\circ} \mathrm{C}$. Finally $\mathrm{FeCl}_{3} \cdot 6 \mathrm{H}_{2} \mathrm{O}$ (Fluka, Milwaukee, WI) was dissolved in this hot solution to make a $0.02 \mathrm{M} \mathrm{Fe}^{3+}$ solution. The beaker was closed and put into an oven that was preheated to $100^{\circ} \mathrm{C}$, where it remained for 1 week. A sample was taken after 1,2,3,5, and 6 days.

The second method deviated from the first in that the $\mathrm{FeCl}_{3} \cdot 6 \mathrm{H}_{2} \mathrm{O}$ was dissolved in doubly distilled water at room temperature to make a $0.5 \mathrm{M} \mathrm{Fe}^{3+}$ solution, in stead of dissolving directly into preheated water. Concentrated $(37 \mathrm{wt} \%) \mathrm{HCl}$ then was directly added to the $0.5 \mathrm{M}$ ferric chloride solution to make the solution $0.025 \mathrm{M} \mathrm{HCl}$. This solution was put into a large beaker and heated to $90-100^{\circ} \mathrm{C}$, while stirring. This solution was diluted with heated doubly distilled water $\left(90^{\circ} \mathrm{C}<T<100^{\circ} \mathrm{C}\right)$ to a factor 25 , to prepare a $0.02 \mathrm{M} \mathrm{Fe}^{3+}$ and $0.001 \mathrm{M} \mathrm{HCl}$ solution. The solution was stirred for a few minutes and then put into two Pyrex glass beakers. These beakers were closed and put into an oven that was preheated to $100^{\circ} \mathrm{C}$, where it remained for 1 day and for 1 week, respectively. The difference here with the first method is that the solution of ferric chloride was not directly made at high temperature $\left(90-100^{\circ} \mathrm{C}\right)$, but was first at room temperature in the form of a concentrated solution $(0.5 \mathrm{M})$. Hydrolysis therefore might already start at temperatures below $90^{\circ} \mathrm{C}$. 


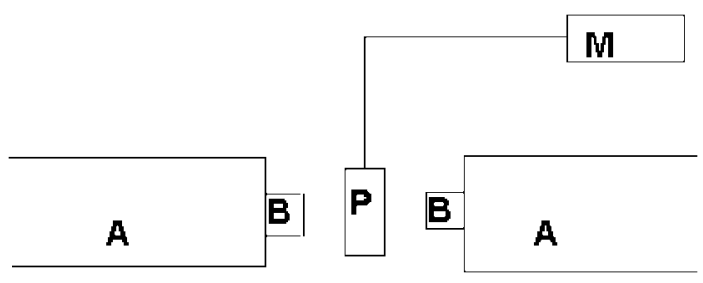

FIG. 1. Setup used for the magnetization measurements. B, a small fluctuating magnetic field is applied between these coils; $\mathrm{P}$, adhesive tape with powder; $\mathrm{M}$, meter that measures the movement of $\mathrm{P}$.

For the third method the $\mathrm{FeCl}_{3} \cdot 6 \mathrm{H}_{2} \mathrm{O}$ (Fluka) was first dissolved in doubly distilled water at room temperature to make a $0.5 \mathrm{M} \mathrm{Fe}^{3+}$ solution. Concentrated $(37 \mathrm{wt} \%) \mathrm{HCl}$ was added to the $0.5 \mathrm{M}$ ferric chloride solution to make the solution $0.025 \mathrm{M}$ in $\mathrm{HCl}$. This solution was diluted with doubly distilled water by a factor 25 , to make a $0.02 \mathrm{M} \mathrm{Fe}^{3+}$ and $0.001 \mathrm{M} \mathrm{HCl}$ solution. The mixture was stirred for a few minutes and than poured into a glass beaker. This beaker was closed and put into an oven that was preheated to $100^{\circ} \mathrm{C}$, where it remained for 1 week. Thus, the difference with method two is that not only the concentrated solution $(0.5 \mathrm{M})$, but also the diluted solution $(0.02 \mathrm{M})$ was made at room temperature, before heating to $100^{\circ} \mathrm{C}$, increasing the chance that precipitation would occur at a temperature below $90^{\circ} \mathrm{C}$.

Dry powder was obtained by centrifuging the suspension until the supernatant was clear, washing the sediment with distilled water, resuspending the particles in distilled water and centrifuging again, and repeating these steps until a dry powder free of chloride was obtained.

Two transmission electron microscope (TEM) instruments were used to investigate the size and morphology of the precipitated particles. Either a Philips EM30 Twin/STEM TEM or a $\mathrm{CM} 10 \mathrm{H}$ Philips TEM was used. In all cases, TEM samples were prepared by addition of alcohol to the dried powder and subsequent dispersion by ultrasonification for $10 \mathrm{~min}$. One drop of the suspension was put on a copper grid that subsequently was dried in normal air prior to use in the TEM apparatus.

For determination of the phase composition and the average crystallite size of the dried powders X-ray diffraction (XRD) and X-ray line broadening (XRLB), respectively, were used. Measurements were performed with a Philips X'Pert-1 PW3710 diffractometer (Eindhoven, The Netherlands), using $\mathrm{Cu} K \alpha(\lambda=$ $1.542 \AA$ ) radiation and a Ni filter. The divergence slit was set to $1^{\circ}$ and the receiving slit to 0.1 . The same apparatus was used to measure the XRLB to determine the average crystallite size of the phases of the powders with the Scherrer formula (7).

The magnetization measurements were performed using a PMC Micro Mag Model 2900 magnetometer. A schematic representation of the apparatus is shown in Fig. 1. Between the two poles (A) a fixed magnetic field of $2 \mathrm{~T}$ is applied. Between the two coils (B) a small fluctuating magnetic field is applied. A small weighed amount of powder is put on adhesive tape $(\mathrm{P})$ and put between the two coils $\mathrm{B}$. The fluctuating magnetic field will cause the magnetic sample $(\mathrm{P})$ to move back and forth according to the change in the magnetic field. At meter (M) the movement of the sample $\mathrm{P}$ is registered. The magnetization for all samples was corrected for the used weight.

\section{RESULTS}

\section{Hydrolysis of Ferric Chloride Solutions}

The moment at which precipitation was first visible was different for the three used varieties of precipitations of a $0.02 \mathrm{M}$ ferric chloride solution. In the second method, with the medium heating rate, precipitation occurred during addition of the concentrated ferric chloride solution. For the third method, with the slowest heating rate, precipitation occurred when heating up the $0.02 \mathrm{M}$ ferric chloride solution from room temperature to $100^{\circ} \mathrm{C}$. If the ferric salt was added directly (the fist method), an orange-red colored suspension resulted after 1 week, while in the other two cases a purple brown suspension was produced.

These differences in observed precipitation behavior were reflected in the TEM pictures and XRD results. Figure 2 shows the particles that were produced if the ferric chloride salt was added directly into the preheated hydrochloric acid solution (method 1). The same particles were present after heating at $100^{\circ} \mathrm{C}$ for 1 day and for 1 week. Regularly shaped hematite crystals of 30 to $60 \mathrm{~nm}$ had been formed. The form was hexagonal to subrounded. XRD revealed the presence of hematite only. The average particle size determined from TEM pictures was $41 \mathrm{~nm}$, while XRLB gave $46 \mathrm{~nm}$ as the average diameter. The size polydispersity of these particles was $15 \%$. This precipitation reaction was repeated several times. The morphology of the precipitated particles was identical for each precipitation, but the average crystallite size, as determined by XRLB, varied from 47 to $71 \mathrm{~nm}$.

If the ferric chloride was not added directly to the preheated hydrochloric acid solution, but first dissolved in cold water before heating to $100^{\circ} \mathrm{C}$ (methods 2 and 3), the results were very

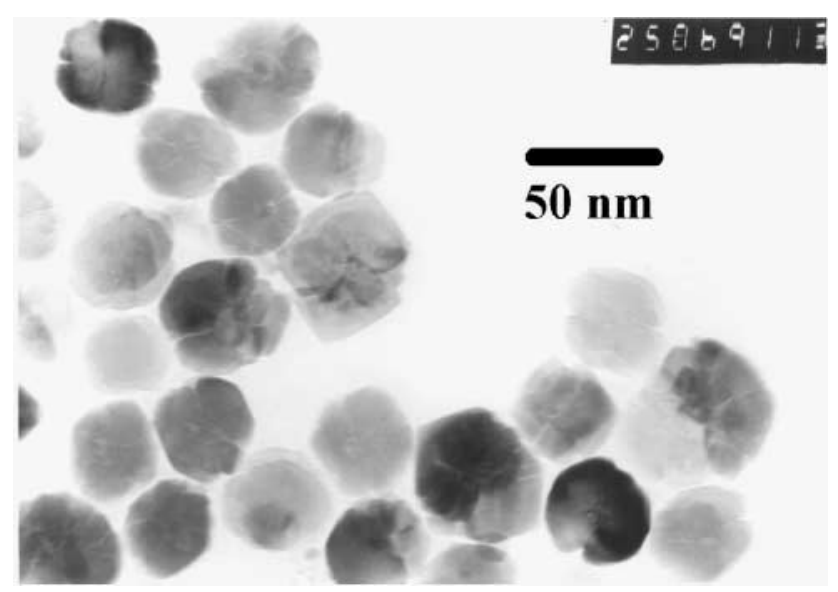

FIG. 2. TEM picture of hematite prepared by hydrolysis of $0.02 \mathrm{M} \mathrm{FeCl}_{3}$ solution made by directly adding the salt at $100^{\circ} \mathrm{C}$, after $1-7$ days at $100^{\circ} \mathrm{C}$. 


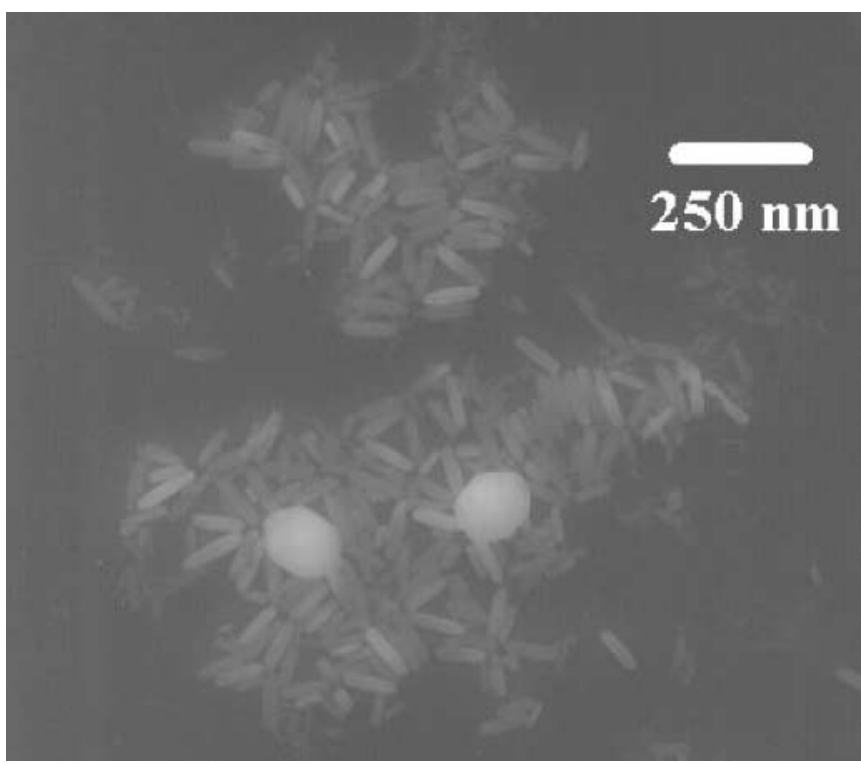

FIG. 3. TEM picture of akaganéite/hematite prepared by hydrolysis of $0.02 \mathrm{M} \mathrm{FeCl}_{3}$ solution after adding $0.5 \mathrm{M} \mathrm{FeCl}_{3}$ to diluted $\mathrm{HCl}$ solution at $100^{\circ} \mathrm{C}$, after 1 day at $100^{\circ} \mathrm{C}$.

different compared to method 1 . The results of method 2 and 3 were similar. Therefore, only the results of method 2 are shown.

A mixture of two particle types was present after 1 day of heating at $100^{\circ} \mathrm{C}$ (method 2), as can be seen in Fig. 3. One type was a spindle of approximately $100 \mathrm{~nm}$ length and $20 \mathrm{~nm}$ width, while the other had an oval shape, with an average length of $150 \mathrm{~nm}$ and width of $100 \mathrm{~nm}$. XRD showed the presence of two phases, hematite and akaganéite. After heating for 7 days at $100^{\circ} \mathrm{C}$, only the oval-shaped particles were left (see Fig. 4),

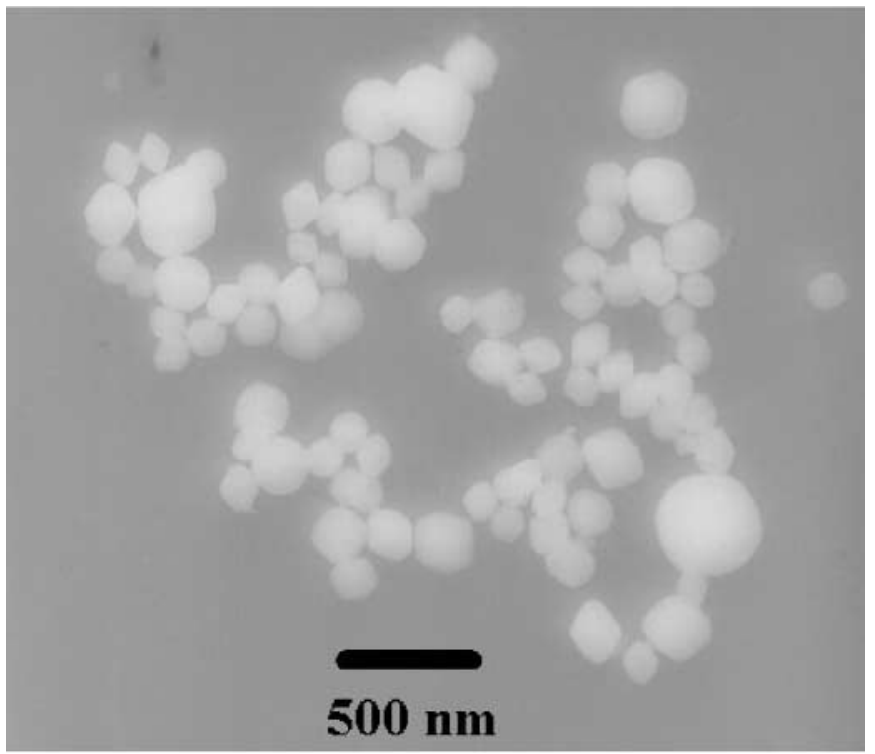

FIG. 4. TEM picture of hematite prepared by hydrolysis of $0.02 \mathrm{M} \mathrm{FeCl}_{3}$ solution after adding $0.5 \mathrm{M} \mathrm{FeCl}_{3}$ to diluted $\mathrm{HCl}$ solution at $100^{\circ} \mathrm{C}$, after 7 days at $100^{\circ} \mathrm{C}$.
TABLE 1

Results of Magnetization Measurements on Hematite Particles Prepared by Hydrolysis of $0.02 \mathrm{M} \mathrm{FeCl}_{3}$ Solutions

\begin{tabular}{|c|c|c|c|c|c|c|}
\hline $\begin{array}{l}\text { Diameter, } \\
\text { TEM (nm) }\end{array}$ & $\begin{array}{l}\text { Polydispersity } \\
(\%)\end{array}$ & $\begin{array}{c}\text { Diameter, } \\
\text { XRLB }(\mathrm{nm})\end{array}$ & $\begin{array}{c}H_{\mathrm{c}} \\
(\mathrm{mT})\end{array}$ & $\begin{array}{c}M_{\mathrm{r}} \\
(\mathrm{nAm})\end{array}$ & $\begin{array}{c}M_{\mathrm{S}} \\
(\mathrm{nAm})\end{array}$ & $M_{\mathrm{r}} / M_{\mathrm{s}}$ \\
\hline 41 & 15 & 47 & 0.51 & 0.67 & 128 & 0.0053 \\
\hline 59 & 13 & 71 & 3.91 & 23.6 & 454 & 0.0519 \\
\hline 160 & 16 & & 108 & 99 & 269 & 0.369 \\
\hline
\end{tabular}

Note. $H_{\mathrm{c}}$, coercive force; $M_{\mathrm{r}}$, magnetization at $H=0 \mathrm{~T} ; M_{\mathrm{s}}$, (maximum) magnetization reached at $H=1.6 \mathrm{~T}$.

which were shown to be hematite by XRD. The majority of these hematite crystals had the same shape and size as after 1 day, but a minority of crystals had grown to larger particles of up to $500 \mathrm{~nm}$ diameter. Both methods 2 and 3 thus led to larger hematite crystals compared to method 1 .

\section{Magnetization of Subrounded Particles}

The magnetization of the subrounded particles made by the hydrolysis of ferric chloride solution was measured as function of particle size. Three powders were used for this. The first two, with the smallest particle sizes, were two different batches of the first precipitation method, the third was a powder prepared by the second precipitation method. The values mentioned in Table 1 were calculated for $1 \mathrm{mg}$ of material for each powder. $H_{\mathrm{c}}$ and $M_{\mathrm{r}} / M_{\mathrm{s}}$ are both measures for the amount of ferromagnetic material present in the sample. The higher $H_{\mathrm{c}}$ and $M_{\mathrm{r}} / M_{\mathrm{s}}$, the more ferromagnetic the material. It is clear that the ferromagnetic nature decreases with particle size, because the anisotropic energy barrier for rotation of magnetic moments is proportional to the particle volume.

In Fig. 5, the magnetization curves of the largest particles of $160 \mathrm{~nm}$ are shown. It is clearly visible that these larger particles

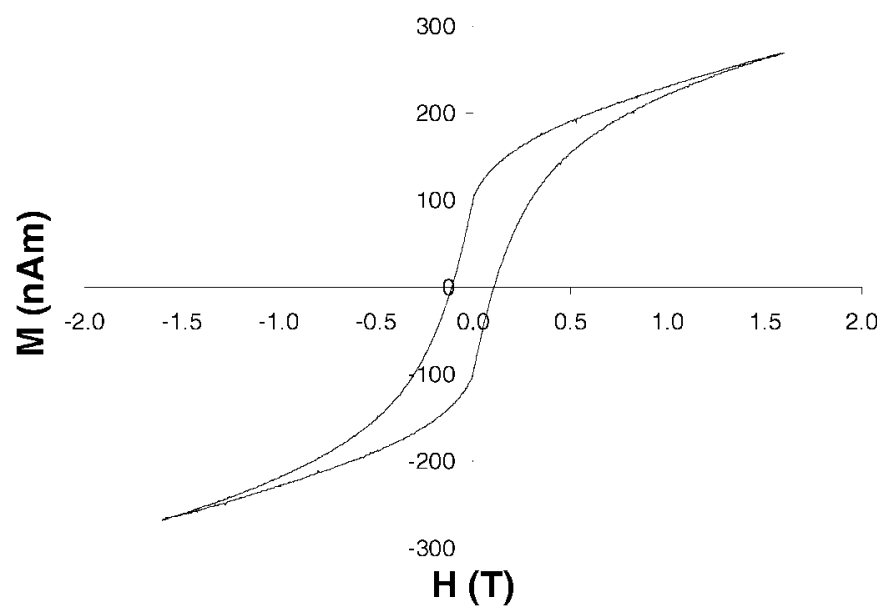

FIG. 5. Magnetization measurement of subrounded hematite particles with $160 \mathrm{~nm}$ average size. 


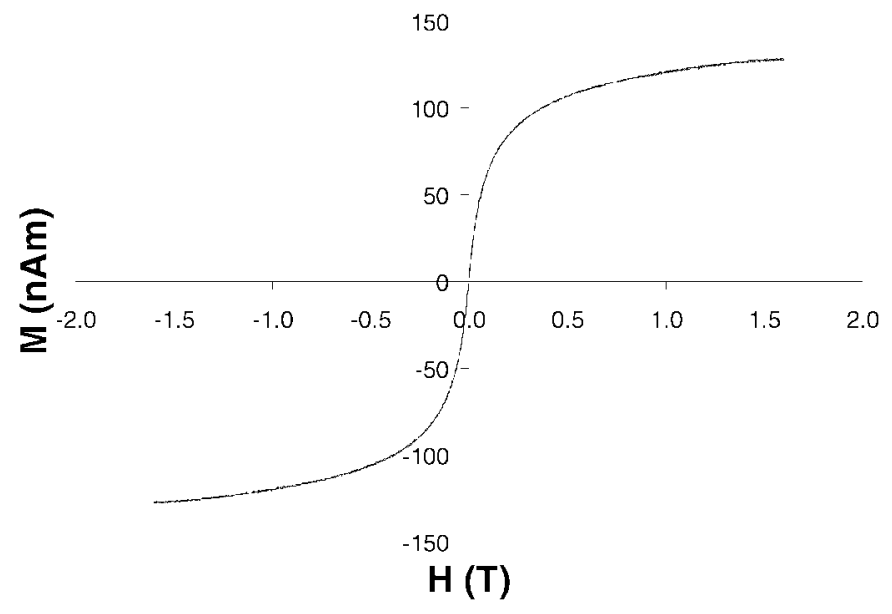

FIG. 6. Magnetization measurement of subrounded hematite particles with $41 \mathrm{~nm}$ average size.

exhibit hysteresis. The hematite particles with average size of $41 \mathrm{~nm}$ have almost no remnant magnetization at zero magnetic field strength, as can be seen in Fig. 6, which is an indication of superparamagnetism.

\section{DISCUSSION}

\section{Hydrolysis of Ferric Chloride Solutions}

The results of the precipitation experiments with ferric chloride show that very small changes in precipitation conditions may cause very large differences in the resulting crystal structure, size, and morphology. Matijevic and Scheiner were the first to report on the hydrolysis of a $0.02 \mathrm{M}$ ferric chloride solution by heating at $100^{\circ} \mathrm{C}$ for 1 week (6). The hydrolysis resulted in subrounded particles with an average size of $46 \mathrm{~nm}$. This hydrolysis was performed by addition of a concentrated (3.0 M) ferric chloride solution to a hydrochloric acid solution (6). In our study the aqueous ferric ion concentration could not be increased to above $0.5 \mathrm{M}$ without the occurrence of precipitation reactions. Therefore, this $0.5 \mathrm{M}$ solution was used to prepare a diluted $(0.02 \mathrm{M})$ ferric chloride solution at $100^{\circ} \mathrm{C}$ (method 2). This seemingly small difference to the method of Matijevic and Scheiner (6) led to very different particles as reported by these authors (6). Much larger hematite particles were formed. This difference can probably be explained by the difference in $\mathrm{pH}$ between the two stock solutions. In the $0.5 \mathrm{M} \mathrm{Fe}^{3+}$-solution hydrolysis of the ferric ions may have occurred that did not occur in the more acidic $3.0 \mathrm{M} \mathrm{Fe}^{3+}$. The lower the ferric chloride solution, the higher the $\mathrm{pH}$ of the solution will be and the faster hydrolysis will occur.

If the prescription of Cornell and Schwertmann (8), who pointed out that the ferric chloride had to be added directly to the preheated $\mathrm{HCl}$ solution, was followed (method 1), the results of Matijevic and Scheiner were reproduced (6). Many investigators have reported on this precipitation reaction, following the description of Matijevic and Scheiner (6). Most obtained the same morphology after heating at $100^{\circ} \mathrm{C}$ for 1 day, and reported average crystallite sizes varying from $46 \mathrm{~nm}$ (9) to $80 \mathrm{~nm}$ (10), just as was found in this study by repeating the same precipitation reaction several times. These deviations from the results of Matijevic and Scheiner (6) are probably caused by small differences in the hydrolysis rate. Bailey et al. (11) reported for the same hydrolysis reaction that only after 7 days spherical particles with an average size of $250 \mathrm{~nm}$ were formed.

Figures 3 and 4 and the XRD results show that before hematite was formed in the solution, akaganéite was formed as was reported before $(6,11)$. At a temperature $>80^{\circ} \mathrm{C}$ a phase transformation to hematite takes place. Below this temperature, goethite is formed (12). The fact that neither method 2 nor method 3 led here to the formation of goethite indicates that any early formed akaganéite only started to transform after the temperature of the ferric chloride solutions (whether diluted or concentrated) was increased to above $80^{\circ} \mathrm{C}$. Thus, the heating went faster than the transformation. Akaganéite is formed faster than hematite (or goethite) since it has a lower energy for nucleation. Hematite is a more thermodynamically stable crystal structure with a solubility in water lower than akaganéite (8).

\section{Magnetic Properties}

The smallest measured hematite particles (41 nm) are apparently single-domain particles, which also exhibit superparamagnetism. This implies that the magnetic domain size of these subrounded hematite particles is approximately or perhaps slightly larger than $40 \mathrm{~nm}$.

As mentioned before, different values are given in literature for the critical particle size for transition of ferromagnetic to superparamagnetic behavior. These differences are related to differences in particle shapes and differences in the amount of strain and defects in the particles. Muench et al. (13) annealed hematite particles with sizes ranging from 30 to $326 \mathrm{~nm}$ at $500^{\circ} \mathrm{C}$. In all cases the annealing led to an increase in $T_{\mathrm{M}}$, the Morin transition temperature. This is the temperature at which hematite changes from canted antiferromagnetic to fully antiferromagnetic. This increase in $T_{\mathrm{M}}$ indicates that annealing makes the hematite less paramagnetic, since paramagnetic hematite would not have a $T_{\mathrm{M}}$. The raising of $T_{\mathrm{M}}$ due to annealing can according to Muench et al. (13) be attributed to the decrease in the amount of lattice defects and strain. These observations are supported by Amin et al. (2). They showed that both $H_{\mathrm{c}}$ and $M_{\mathrm{r}} / M_{\mathrm{s}}$ of ferromagnetic hematite particles increased due to annealing at $300^{\circ} \mathrm{C}(2)$. They reported a value of 0.1 for $M_{\mathrm{r}} / M_{\mathrm{s}}$ for unannealed hematite particles with $40 \mathrm{~nm}$ average diameter (2), which is much higher compared to the here reported value of 0.005 , although the particles were made by seemingly identical synthesis routes. Again, these differences can be explained only by small differences in the synthesis route, probably resulting in differences in the defect structure of the particles. Although annealing normally decreases the amount of lattice strain and defects in a powder, it can also cause coalescence of crystallites, increasing the average 
crystal size. This last effect will certainly strengthen the ferromagnetic nature of nanosized hematite particles, which makes it difficult to separate the influence of the lattice strain and defects from the influence of the crystallite on the ferromagnetic nature.

Kündig et al. reported a critical size of $13.5 \mathrm{~nm}$ for hematite particles made by impregnation of silica with ferric salt solution and subsequent calcination at $500^{\circ} \mathrm{C}$. The morphology of these particles was not described (5). For spherical hematite particles the critical size was reported to be $8 \mathrm{~nm}(3,4)$. The largest critical particle size for the transition from ferromagnetic to superparamagnetic behavior for hematite particles reported so far is $32 \mathrm{~nm}$ (14), which is slightly lower than the here reported value of approximately $40 \mathrm{~nm}$.

Strain in the hematite particles does not seem to be a likely cause for the large critical particle size reported here, since the crystallite size determined by XRLB was $46 \mathrm{~nm}$ for the smallest hematite particles, while TEM showed $41 \mathrm{~nm}$. If the hematite particles would be strained, XRLB should indicate a lower particle size than TEM. The used synthesis methods seems to have produced particles with a well-ordered and unstrained lattice, since both the particles with average size 41 and $59 \mathrm{~nm}$ (as measured with TEM) have larger particle sizes if the XRD data would be taken as a measure.

\section{SUMMARY AND CONCLUSIONS}

Subrounded, uniform, and well-ordered crystalline hematite particles with unstrained lattice were made with an average diameter of $41 \mathrm{~nm}$ and a narrow particle size distribution within one day by hydrolysis of a $0.02 \mathrm{M}$ ferric chloride solution. To obtain these particles it was important to add the ferric chloride directly into the preheated hydrochloric solution $\left(T>90^{\circ} \mathrm{C}\right)$. The hydrolysis of ferric salt solutions is very sensitive to small changes in the reaction conditions, which result in large variations of average hematite particle size and shape. The magnetic behavior of the produced subrounded hematite particles changed from canted antiferromagnetic, as normal for hematite, to paramagnetic when decreasing the particle size from 160 to $41 \mathrm{~nm}$. This is the largest size reported thus far for superparamagnetic hematite particles.

\section{ACKNOWLEDGMENTS}

Gerard van Ewijk and Bonny Kuipers of the University of Utrecht are acknowledged for the measurement of magnetization curves. Daphne Beukers and Jason Vu of the University of Twente are acknowledged for their assistance to the synthesis of hematite particles and particle size determinations. This work was supported by the Foundation for Fundamental Research on Matter (FOM) and the group of Chemical Sciences $(\mathrm{CW})$ which are both parts of the Netherlands Organization for Advancement of Research (NWO).

\section{REFERENCES}

1. Chikazumi, S., "Physics of Magnetism," Wiley, New York, 1964.

2. Amin, N., Arajs, S., and Matijevic, E., Phys. Status Solidi A 104, K65 (1987).

3. Dormann, J. L., Cui, J. R., and Sella, C., J. Appl. Phys. 57, 4283 (1985).

4. Zysler, R., Fiorani, D., Dormann, J. L., and Testa, A. M., J. Magn. Magn. Mater. 133, 71 (1994).

5. Kündig, W., Bömmel, H., Constabaris, G., and Lindquist, R. H., Phys. Rev. 142, 327 (1966).

6. Matijevic, E., and Scheiner, P., J. Colloid Interface Sci. 63, 509 (1978).

7. Scherrer, P., Goett. Nachr. 2, 98 (1918).

8. Cornell, R. M., and Schwertmann, U., "The Iron Oxides: Structure, Reactions, Occurrence and Uses," VCH, Weinheim, Germany, 1996.

9. Zhang, J., and Buffle, J., J. Colloid Interface Sci. 174, 500 (1995).

10. Kallay, N., Fischer, I., and Matijevic, E., Colloids Surf. 13, 145 (1985).

11. Bailey, J. K., Brinker, C. J., and Mecartney, M. L., J. Colloid Interface Sci. 157, 1 (1993).

12. Sugimoto, T., Sakata, K., and Muramatsu, A., J. Colloid Interface Sci. 159, 372 (1993).

13. Muench, G. J., Arajs, S., and Matijevic, E., Phys. Status Solidi A 92, 187 (1985).

14. Takada, T., and Kawai, N., J. Phys. Soc. Jpn. 17, 691 (1962). 